JOSEFINA FERNANDA VIDAL MIRANDA

PONTIFICIA UNIVERSIDAD CATÓLICA DE CHILE

SANTIAGO, CHILE

JFVIDAL@UC.CL
Financiamiento: Consejo Nacional de la Cultura y las Artes, Fondart Nacional, línea de Diseño, modalidad de Investigación, convocatoria 2018.

\section{Paisaje y dinámicas de interacción social en Providencia: moda y prêt-à-porter chileno (1967-1987)}

Landscape and social interaction dynamics in Providencia: fashion and Chilean ready-to-wear (1967-1987)

Resumen. La llegada de la forma de producción conocida como prêt-à-porter, desde Europa a un sector de la capital de Chile, tuvo como consecuencia un proceso de adaptación de la tendencia internacional al medio local. El presente artículo busca ahondar en los factores identitarios del prêt-à-porter chileno a partir del análisis de los métodos de producción, comercialización y difusión de un conjunto de boutiques ubicadas en la zona Oriente de Santiago, que fueron las primeras oferentes de estas novedosas prendas de vestir. Estos enclaves definieron una geografía de la moda en el sector comercial de la comuna de Providencia, que se caracterizó por facilitar el recorrido a pie de sus visitantes. Eventualmente, esta cartografía de la moda se dispersó a medida que la economía de libre mercado fomentó la llegada del mall y sus herméticas dinámicas de compra.

Palabras clave: identidad, industria textil chilena, manufactura chilena, prêt-à-porter.

Abstract. The arrival of a form of production known as ready-to-wear, coming from Europe to an area of the capital of Chile, resulted in an adaptation process of the international trend to the local milieu. This article seeks to delve into the identifying factors of Chilean ready-to-wear by analyzing the modes of production, commercialization, and dissemination of a group of boutiques located in the eastern part of Santiago that were the first offerers of the novel garments. These settlements defined a geography of fashion in the commercial area of the commune of Providencia that facilitated going through it by foot. Eventually, this walkable fashion cartography started to dissolve as the free market economy encouraged the arrival of the mall and its enclosed dynamics.

Keywords: boutique, Chilean garment industry, Chilean manufacturing, consumer culture history, geography of fashion, identity, ready-to-wear

Fecha de recepción: 30/07/2018

Fecha de aceptación: 12/09/2018

Cómo citar: Vidal Miranda, J. (2018) Paisaje y

dinámicas de interacción social en Providencia:

moda y prêt-à-porter chileno (1967-1987).

RChD: creación y pensamiento, 3(5), 1-13

DOI: $10.5354 / 0719-837 X .2018 .50728$

Revista Chilena de Diseño,

RChD: creación y pensamiento

Universidad de Chile

2018, 3(5)

http://rchd.uchile.cl 
1. Este marco temporal se inicia con la instalación de Tai y Vog, dos de las boutiques más importantes dentro de la escena capitalina local en ese entonces. Además, en 1967 se comenzó a editar la revista femenina Paula, donde se publicaron las primeras fotografías de moda realizadas en Chile, lo que significó el inicio de la construcción de un imaginario en torno a la moda nacional. Se escoge el año 1987 como punto de término de la periodización de este proyecto en coincidencia al cierre de la emblemática tienda de ropa Palta, lo que en alguna medida fue un indicador del declive de este sistema de boutiques y pequeños enclaves de la moda e indumentaria en la Región Metropolitana.

\section{Introducción}

Entre 1967 y $1987^{1}$, aproximadamente, coexistieron diversos agentes y factores que dieron pie a la instalación de un sistema de la moda en Chile (Montalva, 2015, p. 71), el cual estuvo inscrito en la fórmula del denominado prêt-à-portery representado en una red de boutiques y tiendas minoristas de vestuario ubicadas principalmente en la comuna de Providencia.

Uno de los factores primordiales que permitieron el asentamiento de la red de boutiques en la capital fue el auge en los años sesenta de la industria textil nacional. Esta proporcionó la materia prima necesaria a quienes lideraban las pequeñas tiendas denominadas "boutique". Si bien la indumentaria comercializada en el periodo poseía una fuerte influencia extranjera, casi la totalidad de los modelos eran confeccionados con telas fabricadas en Chile y manufacturados en talleres locales (Vidal, J., \& Álvarez, P., 2018).

Además de una industria productora de géneros y material textil, el país también contó con un grupo de técnicos y expertos capaces de sostener los ritmos de producción de esta nueva oferta de modas. La autora Pía Montalva, en su libro Morir un poco: Moda y sociedad en Chile 1960-1976, se refiere a las nuevas instancias que surgen durante el gobierno de Eduardo Frei Montalva, como las escuelas técnicas del Ministerio de Educación, el Instituto de Promoción del Trabajo y los centros de madres, las cuales permitieron profesionalizar los oficios asociados a la manufactura de indumentaria. En todas estas instituciones, el Estado participa activamente, así concluye Montalva: "Las políticas de promoción popular implementadas bajo el gobierno de Frei Montalva cruzan de distintas maneras este giro desde la alta costura hacia prêt-à-porter, fomentando alternativas laborales insospechadas para mujeres de estratos sociales medios y bajos" (2015, p. 73).

La década de 1960 a nivel mundial se caracterizó -entre otros sucesospor la efervescencia social, agitación y revoluciones culturales y sociales. Es en esta época cuando los jóvenes y las mujeres se transforman en sujetos históricos protagonistas y encuentran mayores instancias de participación pública. En consecuencia, la sociedad chilena también experimentó transformaciones que permitieron una creciente visibilidad para ambos actantes, e incluso tuvieron una forma de expresión en la moda y en la emergencia del prêt-à-porternacional. Ellos fueron quienes dotaron a la moda de nuevos aires y se convirtieron en personajes fundamentales para el desarrollo del fenómeno de las boutiques en el sector Oriente de la capital, al implicarse en modernas dinámicas de consumo e interacción social, antes desconocidas en el medio nacional.

La suma de estos factores permitió la llegada de esta moderna forma de producción al país, que al instalarse en la capital adquirió características singulares de acuerdo a los códigos locales. Conforme a los postulados de Montalva, es en la discrepancia entre el original y la copia donde se constituye el componente identitario del prêt-à-porter chileno (2004, p. 12). En esta línea, este trabajo busca profundizar en el análisis de los métodos de producción, comercialización y difusión de las boutiques en estudio y así determinar rasgos característicos de esta escena precedente de la moda nacional. Asimismo, esta investigación sostiene que parte de la identidad del prêt-àporter chileno se constituye en las interacciones de actantes humanos y no humanos (Latour, 1998) en el territorio de compra. En ese sentido, este trabajo propone la definición de una cartografía de la moda en el sector comercial de Providencia, la cual en su configuración urbana resultó determinante en las 
dinámicas de consumo. Apoyándonos en John Potvin: "Los encuentros con la moda suceden dentro de un espacio, en un lugar determinado y no funcionan simplemente como telones de fondo, sino que son fundamentales para el significado y la vitalidad que trazan las experiencias de la moda" (2009, p. 1). Este trabajo busca ser un aporte a la historia de la cultura del consumo en Chile. Desde esta mirada, la presente investigación analiza cómo se construye una identidad local del prêt-à-porter desde las dinámicas de consumo en el centro comercial de Providencia (Miller, 1998). La escena aquí examinada es anterior a la instalación del primer mall en la capital; asunto que se torna relevante en el panorama actual, donde los malls y las empresas de retail forman parte del imaginario citadino y se han convertido en uno de los principales escenarios urbanos de la vida cotidiana (De Simone, 2015, p. 20). El presente artículo se adentra en la investigación "Moda al paso: Historia de las boutiques y pequeñas tiendas de vestuario de la zona Oriente de Santiago 1967-1987"; concretamente, en los hallazgos que dan cuenta del proceso de resignificación del modelo internacional del prêt-à-porter de acuerdo con la situación y las prácticas nacionales, y de la configuración de una cartografía urbana de la moda en el sector comercial de la comuna de Providencia. En dicho estudio, se recurrió a una metodología de investigación cualitativa, que consistió en producir información que se articuló principalmente mediante la consulta de fuentes primarias. Cabe mencionar que, a la fecha, solo dos autores (Montalva y Salinas) han tratado tangencialmente el fenómeno del prêt-à-portery las boutiques en la capital. En consecuencia, se contempló la realización y el análisis de veintisiete entrevistas estructuradas y semiestructuradas a expropietarios de boutiques, consumidores de moda del periodo y expertos en el tema, en tanto informantes. Además, se revisaron revistas femeninas de la época, consideradas como fuentes primarias escritas y visuales. En este sentido, se estudiaron reportajes pertinentes al caso de estudio y además se recopilaron y analizaron visualmente más de 500 fotografías. Por último, se realizó una cartografía del sector de compras, la cual permitió visualizar la información respecto al uso del espacio urbano.

\section{Textiles chilenos, material de confección del prêt-à-porter nacional}

Ya entrado el siglo XX, y luego de la Gran Depresión ocurrida en 1930, el panorama nacional en materias de comercio e industria dio un importante vuelco. Tras la crisis financiera, la respuesta del Estado chileno ante el riesgo económico fue fomentar "el desarrollo hacia dentro" mediante una política de industrialización por sustitución de importaciones, también llamada ISI. Esta aspiración se materializó en 1939 con la creación de la Corporación de Fomento de la Producción (CORFO), institución pública que tuvo como objetivo desarrollar armónicamente todos los rubros productivos a lo largo del país. Estas nuevas medidas económicas impulsaron la consolidación de una industria textil moderna, donde surgieron decenas de fábricas - la mayoría en manos de inmigrantes árabes e italianoscomo Yarur Manufacturas Chilenas de Algodón, Manufacturas Sumar, Caffarena, Sociedad de Linos La Unión, Moletto Hermanos, entre otras. A la producción de fibras naturales como el algodón, la lana y el lino se sumó la elaboración de fibras sintéticas, como el rayón y el nailon. A fines de la década de 1950 e inicios de la siguiente, la industria textil vivió su mayor época de gloria, generando una gran cantidad de empleos y logrando abastecer cerca del 95\% de la demanda nacional (CORFO. División de Industrias Textiles y de Vestuario, 1974; Frías, 1987).

El apogeo experimentado por el rubro durante los años sesenta fue uno de 
los principales factores que permitió la instalación en Santiago de una red de boutiques y tiendas especializadas de vestuario inscritas en el prêt-à-porter. Los comerciantes que abrían sus boutiques contaban con material textil de buena calidad y de fácil acceso para la confección de sus prendas de ropa, lo que sin duda facilitaba la iniciativa de emprender un negocio en el área del vestuario. Además, las boutiques de mayor renombre y éxito en ventas, como Vog o Miss Paula, tenían una estrecha relación con las fábricas textiles, llegaban incluso a influir en su producción al encargar estampados especiales y exclusivos para la confección de indumentaria de la tienda. Sobre este cercano vínculo, Carmen Rojas, socia de la boutique Vog, comenta: "Nosotras le comprábamos a Comandari en esa época, que era una maravilla y nos hacía géneros especiales. Jorge Comandari era genial, estaba a la vanguardia. Ni en París comprabas telas iguales" (comunicación personal, 4 de abril de 2017). Pese a este período de auge de la industria textil nacional, el funcionamiento de las fábricas se desarrolló por décadas en medio de una fuerte pugna política a nivel país; situación que se vio agravada cuando durante el gobierno liderado por Salvador Allende se tensionó a estos enclaves productivos con el traspaso de las fábricas textiles al Área de Propiedad Social. Las dos empresas más grandes en ese entonces, Yarur y Sumar, no permitieron ser estatizadas, sin embargo, fueron "tomadas" por sus mismos trabajadores (P. Sumar, comunicación personal, 4 de mayo de 2018). Al adoptar estas medidas, la administración socialista formalizó su promesa de acabar con los llamados "monopolios industriales" y, a su vez, cumplía con el compromiso de asignar un rol protagónico a los trabajadores en proceso de estatización, concediéndoles espacios en la administración de las fábricas intervenidas. Esto provocó un gran conflicto social entre la clase trabajadora y los propietarios de las empresas textiles, además de una considerable baja en la cantidad y la calidad de producción. Esta situación se mantuvo así hasta la escisión del 11 de septiembre de 1973, cuando las empresas que habían sido tomadas fueron devueltas a sus originales dueños. El retorno estuvo caracterizado por millonarias deudas del período anterior y por la presencia de interventores militares, quienes poseían las facultades de objetar cualquier tipo de decisión sobre el funcionamiento de las fábricas, sin tener los necesarios conocimientos. Así da cuenta Philip Sumar, de Manufacturas Sumar:

Nos dejaron interventores militares durante mucho tiempo metidos adentro. No entendían nada, era un detrimento la situación. Había que aceptarlo, no teníamos alternativa [...] El interventor podía, en su momento, objetar cualquier cosa; cualquier compra, tenían mucho involucramiento al principio (comunicación personal, 4 de mayo de 2018).

Sumado al control y la vigilancia por parte de los militares a las empresas textiles, el golpe de Estado trajo consigo la puesta en marcha de una nueva política económica. A partir de 1975, con la instalación del modelo neoliberal, el régimen proteccionista que había facilitado la consolidación de la industria nacional se debilitó, y así también el conjunto de fábricas textiles que había dominado el rubro en las décadas anteriores. Los primeros años de apertura económica no significaron grandes dificultades, pero la crisis del dólar en 1982 devino en un grave endeudamiento de las empresas, lo que trajo como consecuencia el cierre de gran parte de las fábricas textiles algunos años más tarde. 


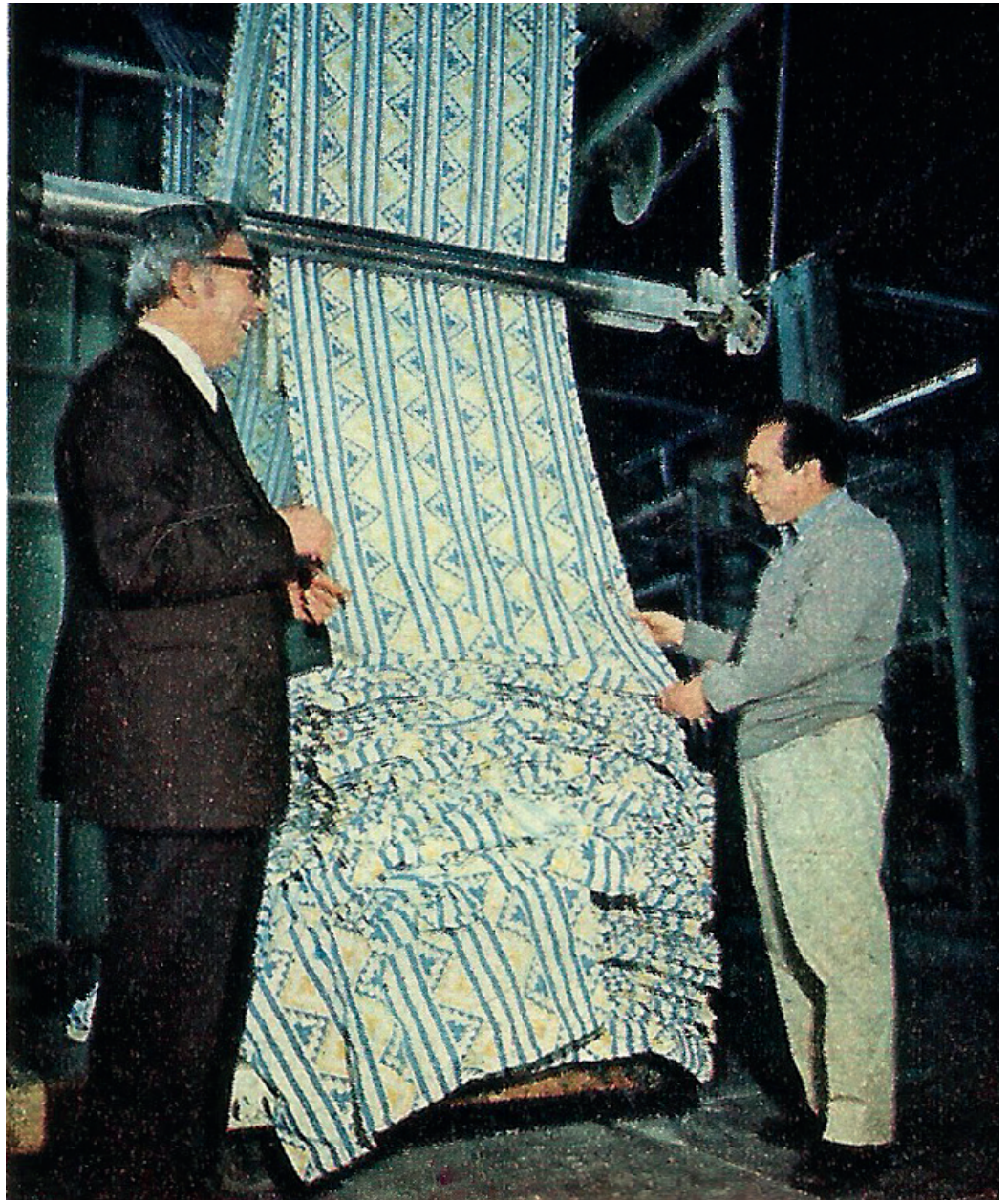

\section{Prêt-à-porter a baja escala}

El término prêt-à-porter es una expresión de origen francés que puede traducirse como "listo para llevar" y hace referencia a las prendas de moda producidas en serie con patrones que se repiten dependiendo de la demanda. La importación de este modelo al medio nacional dio cuenta de un proceso de resignificación de las tendencias internacionales. Esto significó ceñir la lógica del prêt-à-porter de "producir industrialmente vestidos accesibles para todos" (Lipovetsky, 2009, p. 122) a una escala local.

Las personas que lideraron estos negocios que produjeron y comercializaron por primera vez prêt-à-porter en el medio local eran en su mayoría mujeres provenientes de las clases acomodadas, las que, confiadas en su buen gusto, importaban tendencias desde los centros de la moda al territorio nacional y delegaban la producción a talleres locales. Muy pocos de aquellos que se aventuraban con la instalación de una boutique poseían estudios previos relacionados con el diseño de vestuario o conocimientos técnicos de corte y confección, salvo algunas excepciones. A decir verdad, para muchas de las boutiques nacionales el proceso de creación de las prendas se basaba en una copia e imitación de modelos extranjeros (Vidal, J., \& Álvarez, P., 2018). Marta Echaurren, integrante de la boutique Shock, es radical en sus aseveraciones
Figura 1. La toma de los trabajadores en la fábrica Yarur Manufacturas Chilenas de Algodón tuvo como una de sus consecuencias que el arquitecto Enrique Concha diseñara una línea de estampados inspirada en la cultura Diaguita. En la imagen, Vicente Poblete, el interventor, junto a Juan Carvajal, jefe de producción. Fuente: Revista Paloma, núm. 2, 1972. 
2. María Inés Solimano fue parte del proyecto

denominado "Moda Autóctona", un término que surge en 1968 a raíz de una entrevista publicada en la revista Paula a Marco Correa, diseñador de la boutique Tai. El título de la entrevista anunciaba: "Un chileno diseñó la moda latinoamericana." La Moda Autóctona se caracterizó por rescatar elementos típicos del territorio chileno; materiales, formas gráficas, colores, etc. y aplicarlos al ámbito textil y de vestuario, distanciándose de esta manera de las tendencias foráneas. Sus máximos exponentes fueron: Marco Correa, María Inés Solimano, Nelly Alarcón, Enrique Concha y Alejandro Stuven cuando señala: "para qué vamos a diseñar, cuando de ninguna manera podemos competir con los europeos" (Montalva, 2004, p. 80). En la misma línea ideaba sus creaciones la tienda Vog, la cual se caracterizó por implantar en el medio local las tendencias que se imponían en Europa. Así lo deja entrever Carmen Rojas: “Después de ir a Europa, me di cuenta que la Brigitte Bardot usaba unos vestidos cortos tejidos. Entonces, yo los empecé a hacer aquí y tuvieron mucho éxito" (comunicación personal, 4 de abril de 2017). Si bien la copia y adaptación de las tendencias europeas y estadounidenses era el patrón que se repetía en el ámbito de la moda local, existieron ciertos creadores con pretensiones distintas, quienes más allá de vender la novedad, se dedicaban al desarrollo de diversos oficios, ya fuera el tejido, el bordado, entre otros. En esta línea, destaca el trabajo de Laura Rivas y María Inés Solimano². Otra de las singularidades del prêt-à-porter chileno es que lejos de ser un fenómeno accesible a las masas como lo fue el consumo del ready-to-wear en Londres -específicamente en las calles Carnaby Street y King's Road- este se caracterizó por la exclusividad de su oferta, procedente del reducido número de prendas por modelo confeccionadas y expuestas en la tienda. En consecuencia, el prêt-à-porter nacional estuvo en permanente contacto con la alta costura, era ropa hecha a medida. Por lo general, las boutiques exhibían una o dos tallas por prenda, las cuales se ajustaban al cuerpo de los clientes. Así lo indica Luis Eduardo Covarrubias, propietario de la ex boutique Wales, ubicada en la galería comercial Drugstore: "Teníamos una talla de tienda estándar tipo 42 [...] el taller estaba ahí mismo en la tienda, venía el cliente, se probaba y se le ajustaba si era necesario" (comunicación personal, 3 de abril de 2017). Tras la manufactura en talleres de vestuario locales, eran los mismos propietarios de las boutiques quienes comercializaban sus productos. La venta de prendas de ropa no era un trabajo relegado a terceros, sino que exigía que los dueños estuviesen en la tienda a la espera de la posible llegada de un cliente. Asimismo, eran ellos quienes articulaban las dinámicas con los clientes y entre clientes, además de que eran considerados expertos en materia de moda y tendencias. En consonancia, Rojas afirma: "Si no estábamos no iban. Cada salida mía significaba perder $\$ 400.000$ porque la gente entraba y si no estaba en ese momento, no se mandaban a hacer el vestido. Era absolutamente personalizado" (comunicación personal, 4 de abril de 2017). Esta moderna oferta de vestuario manufacturada en territorio nacional y comercializada con una dinámica de venta personalizada coincidió con un detonante crecimiento de la industria cultural, el cual permitió divulgar el trabajo llevado a cabo por las boutiques y tiendas de vestuario recién instaladas, a través de diversas manifestaciones. La difusión de estos negocios se articuló principalmente en torno a dos ejes: los desfiles y las fotografías de moda publicadas en revistas femeninas nacionales. Los desfiles de moda no fueron un fenómeno impuesto en los años sesenta, sino que se habían comenzado a desarrollar de manera sistemática en Chile a fines de la década del cuarenta. Estos eventos mantuvieron, en mayor o menor medida, su carácter de reunión social, donde lo central no siempre eran las prendas de vestir, sino las relaciones y vínculos sociales que permitían la participación en estos acontecimientos. Las producciones de moda en las publicaciones femeninas funcionaban con una dinámica similar, ya que por lo general las dueñas de las boutiques conocían a quienes trabajaban en las revistas ${ }^{3}$ o bien, pertenecían al mismo círculo social. 


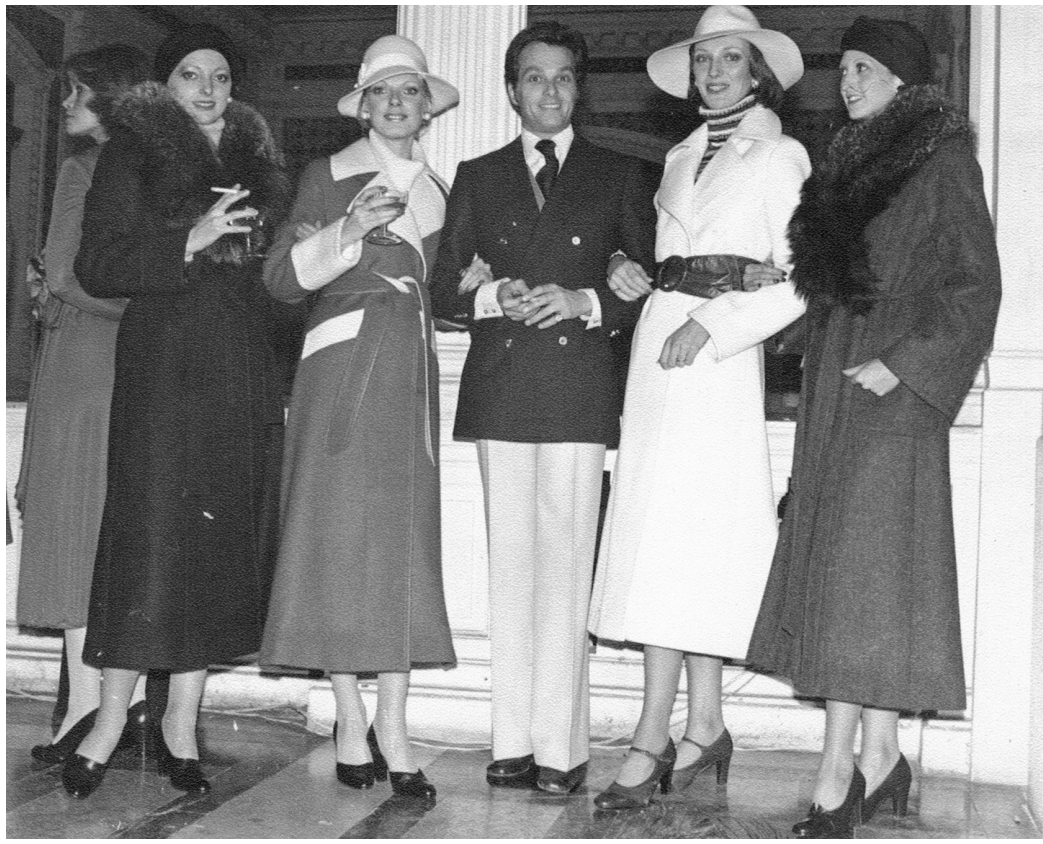

Las publicaciones magazinescas no solo prestaban vitrina a las creaciones de las boutiques, sino que también influían en su producción. En variadas ocasiones, sucedía que las boutiques no ofrecían vestuario que se ajustase a las temáticas de las fotografías, entonces las productoras de las fotografías de moda de las revistas les encargaba confeccionar ciertas prendas específicas para sus fotos. Así lo corrobora Constanza Vergara, ex directora de la revista Paula (1975-1983): "Muchas veces Paula no tomaba en cuenta la ropa que las tiendas tenían, sino que les imponíamos ciertas tendencias [...] decidíamos, por ejemplo, hacer unas fotos de faldas midi y si las boutiques no tenían, debían hacerlas si querían aparecer publicadas" (C. Vergara, comunicación personal, 24 de abril de 2017).

\section{La configuración de una cartografía de la moda en Providencia}

Para adentrarnos en el fenómeno que atañe a esta investigación, es necesario conocer la expansión urbana y comercial de Santiago desde la segunda mitad del siglo XX, la que favoreció a una descentralización del triángulo fundacional de la ciudad. Para fines de los años sesenta, existía un subcentro ubicado en el sector de la avenida Providencia con Ricardo Lyon. Este nuevo espacio urbano se conformó como producto del desplazamiento, desde la década del cuarenta, de la clase media capitalina hacia el sector Oriente, que además detonó la iniciativa del gobierno de Eduardo Frei Montalva de comenzar la planificación para el trazado de la Línea I del Metro de Santiago (Mora y Zapata, 2004). Paralelamente a la construcción de la Línea ı, Providencia inició en 1967 los estudios preliminares para llevar a cabo una normativa comunal que regulara la instalación del comercio en el sector. Germán Bannen, asesor urbanista de la comuna de Providencia, buscó conjugar los nuevos asentamientos privados con la construcción de espacios públicos para los transeúntes, evitando la instalación de edificios comerciales "segregados de los espacios de la ciudad" (Schlack, 2001, p. 20).

Es en este nuevo espacio de consumo, "un lugar construido para el tiempo

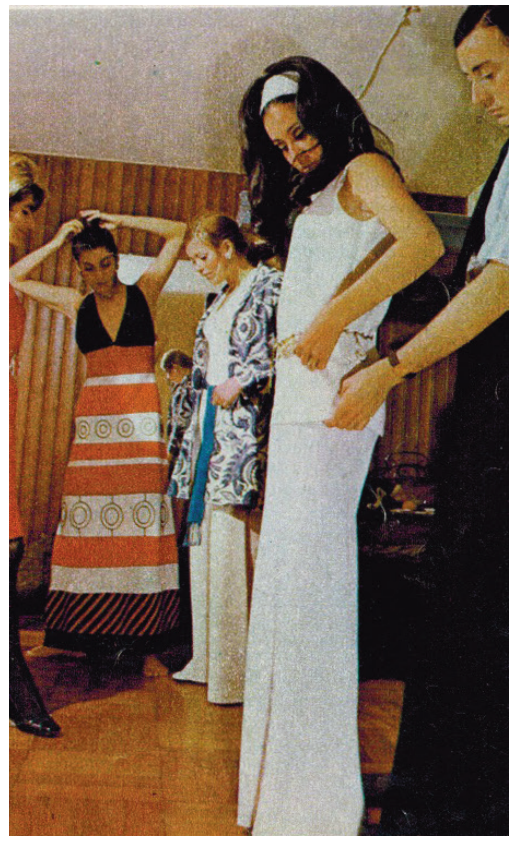

Figura 2. Desfile de Wales en 1972 en el Palacio Cousiño. Fuente: Archivo personal Luis Eduardo Covarrubias.

Figura 3. En la imagen una dinámica habitual, el propio diseñador de la boutique Tai Marcos Correa y Rebeca Izquierdo, una de las tres socias, probando los últimos modelos de la tienda. Fuente: Revista Eva, N¹283, 1969.

3. La revista más relevante y que articuló un quiebre respecto a las publicaciones anteriores sobre temas de moda fue Paula. Esta publicación se lanza en 1967 y desde su tercer número se incluyen periódicamente fotografías de moda producidas en Chile. Estas primeras imágenes dieron inicio a la construcción de un imaginario en torno a la moda nacional, con producciones y escenarios propios. A la luz de este suceso Eva, que se publicaba en Chile desde 1942, renovó su equipo en 1968 con el objetivo de darle aires nuevos a la revista, y comenzó a producir contenido y fotografías de moda en el país. 
Figura 4. Artículo escrito por Germán Bannen para la revista CA, donde explica la implementación del Plan de Pasajes Peatonales. Fuente: revista $C A, \mathrm{~N}^{\circ} 27,1980$

4. El término francés flâneur significa paseante o callejero. El autor Charles Baudelaire usó la palabra en sus poemas y posteriormente Walter Benjamin la convirtió en una figura literaria académica. Con flâneur, Benjamin se refiere al espectador urbano, personaje propio de la vida moderna.
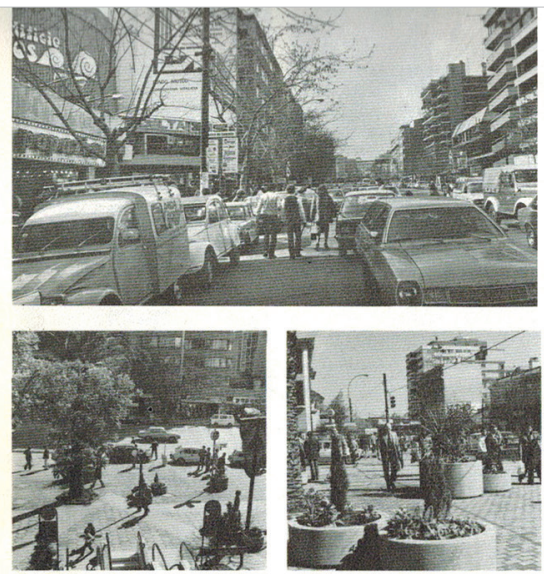

\section{PROVIDENCIA, UNA CALLE DE LA CIUDAD} German Bannen L.
Arquitecto.

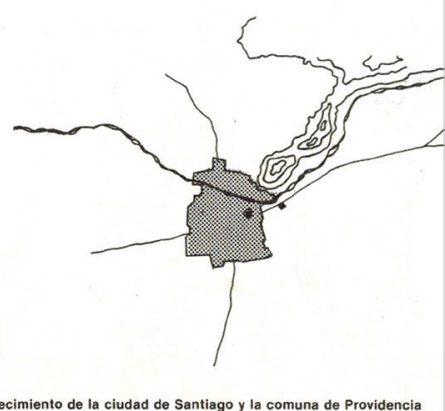

Gล $27 / 28$

del peatón" (Bannen, 1993, p. 34), donde se comenzó a configurar una red de boutiques y tiendas minoristas de moda hacia mediados de los sesenta. La presente investigación propone definir un paisaje comercial, delimitado por la ubicación de estas modernas boutiques localizadas, en su mayoría, en la avenida Providencia entre las calles Pedro de Valdivia y Los Leones, las cuales fueron las primeras oferentes del prêt-à-porter en Chile.

Este terreno de compras acotado y de carácter caminable permitió que las boutiques no fueran solo espacios de consumo y acceso a las últimas tendencias en moda, sino que además estuvieran significadas por dinámicas de encuentro e interacción social. Las boutiques instaladas en el centro comercial de Providencia se localizaron muy cerca unas de otras, lo que facilitó el recorrido a pie de sus visitantes. Así, la calle fue reforzando su condición de lugar y no se consideró únicamente como un espacio de tránsito (Dussaillant, 2011, p. 334). Gran parte de los entrevistados se refirió a los días sábado por la mañana en Providencia; cuando una multitud de gente se congregaba y recorría las calles como un flâneur, lo que relegaba el acto de consumir a un segundo plano. Así lo testimonia Carlos Pérez, propietario y diseñador de la tienda Zokalo a fines de los años setenta:

Era una época muy efervescente en la moda misma. Yo debo haber tenido 18 o 20 años, el plan era ir al Coppelia un sábado en la mañana, disfrazado lo más estrambótico que pudieras, todos con el pelo largo, había toda una cosa colectiva (C. Pérez, comunicación personal, 5 de octubre de 2016) 


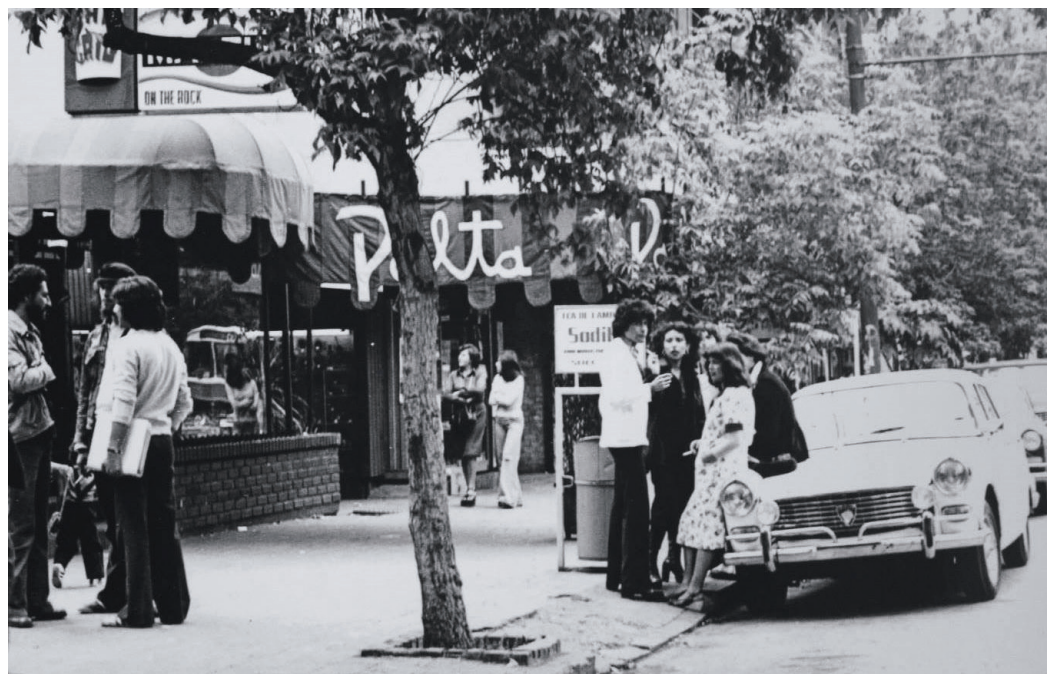

Ricardo Lavín, diseñador que actualmente trabaja con Luis Eduardo Covarrubias en su tienda, evoca que los días sábado, más que un día de compras, eran un día de encuentro y vida social:

Yo me encontraba con mis amigos en Palta, también íbamos mucho a Juvens porque los sábados se acostumbraba a ir, incluso te comprabas la tenida para salir. Mucho rato estábamos en Juvens conversando y no comprando [...]. Había ese concepto de boutiques donde te podías quedar parado, haciendo nada en la tienda. Te encontrabas con tu amigo y a nadie le importaba o el dueño te conocía, y daba lo mismo. Yo creo en ese sector y en esos tiempos había un mundo de caminar solamente (R. Lavín, comunicación personal, 3 de abril de 2017).

\section{Nuevas formas de comprar}

Las reformas relacionadas al sistema neoliberal -implementadas en Chile durante las décadas de 1970 y 1980- significaron, en términos económicos, políticos y sociales, la proyección de una nueva manera de afrontar el crecimiento y el desarrollo del país y su sociedad. En consecuencia, las nuevas pautas de la economía modificaron notablemente dos aspectos que están íntimamente relacionados con el objeto de estudio: los hábitos relacionados al consumo y, por otro lado, la expansión de la ciudad de acuerdo a un urbanismo mercantilizado.

Desde mediados de la década de 1970, emergen distintos subcentros urbanos en el sector Oriente de la capital, los cuales fueron definidos por aglomeraciones de comercio y servicios. En el contexto de una dictadura con afanes modernizantes, estos lugares se configuraron de acuerdo a las nuevas necesidades exigidas por sus consumidores. Una de las grandes diferencias de estos emergentes centros comerciales y los anteriores (centro de Santiago y Providencia) fue que la ubicación excéntrica dejó de ser un inconveniente frente a la ausencia de público peatonal y el reconocimiento de un nuevo cliente motorizado (De Simone, 2015, p. 173).

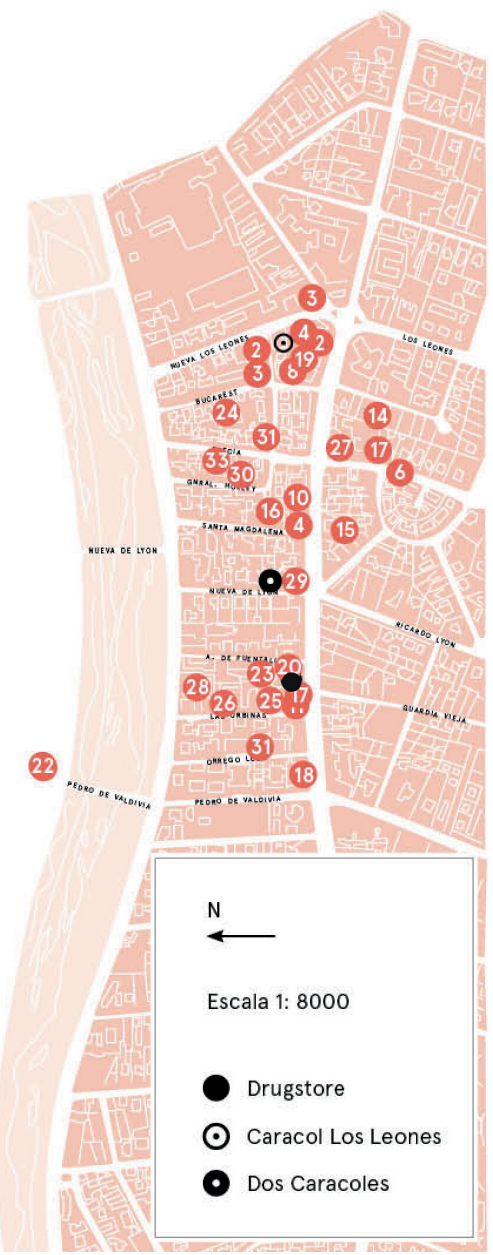

Figura 5. Fotografía tomada por José Moreno Fabbri en 1971. En la imagen se retrata uno de los puntos neurálgicos del centro comercial de Providencia, la tienda Palta y el Coppelia. Fuente: Flickr Santiago Nostálgico.

Figura 6. Mapa del sector de compras donde se señala la ubicación de las boutiques existentes entre 1967 y 1987. Los números se refieren a una tienda en particular. Para ver el detalle consultar las páginas 184-195 del documento disponible en: https://issuu.com/josefinavidalmiranda/docs/ moda_al_paso__osefina_vidal.

Fuente: Elaboración propia. 
Figura 7. El centro comercial de Providencia fue lugar de algunos desfiles de la moda de la época. Uno de ellos fue el innovador desfile de la boutique Sabe, en el centro comercial de Providencia. Para la ocasión, Mercedes Mena, dueña de la tienda, convenció al alcalde para cerrar Av. Providencia desde Lyon hasta Pedro de Valdivia. Además, se consiguió organilleros que musicalizaron el evento y bicicletas Oxford, que fueron facilitadas por quien las fabricaba, a cambio de promocionarlas

Fuente: Archivo personal Mercedes Mena.

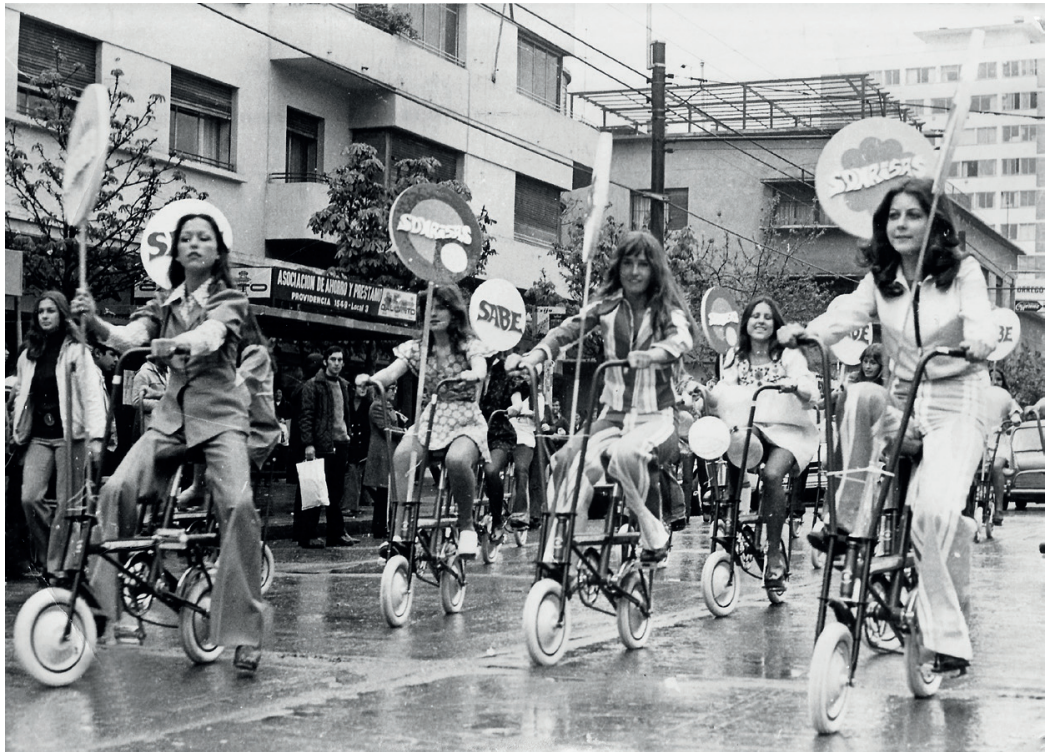

En 1980, el arquitecto Jaime Bendersky, junto a la cadena norteamericana Sears y un grupo de inversionistas brasileños, decide construir el primer mall comercial, una tipología inédita en espacios dedicados al consumo nacional. Después de dos años de obras y enfrentados a una gran crisis económica, se inauguró el Parque Arauco, que ofrecía los horarios de atención más extendidos del comercio de la época, incluso los días domingo. Este se emplazó en plena avenida Kennedy aprovechando su accesibilidad vehicular y ofreció amplios estacionamientos por sus cuatro costados. En su interior se encontraban dos tiendas ancla: Gala Sears y Muricy, aunque estas marcas no tuvieron éxito ya que no se adaptaron al gusto local (M. Mir, comunicación personal, 2 de octubre de 2016).

Pese a la llegada de estas nuevas tipologías de consumo a principios de la década del ochenta, el centro comercial de Providencia continuaba siendo el lugar predilecto de compras para quienes seguían las tendencias en vestuario impuestas por las publicaciones femeninas y los nuevos shows de moda transmitidos por televisión. Las pioneras marcas de retail no significaron una alternativa atrayente para los consumidores de diseño de vestuario en la capital. El mismo año que el Parque Arauco se inauguró (1982), el cuadrante de General Holley y Suecia vivía un periodo de auge, lo que se manifestó en la llegada al sector de los modistos de alta costura nacionales entre 1983 y 1984 , Rubén Campos y Atilio Andreoli. Por otra parte, las dinámicas de compra que ofrecía el centro comercial de Providencia eran muy distintas a las de los shopping centers y el primer mall de Santiago, ambos ubicados en la periferia, los cuales eran espacios cerrados que no poseían conexión alguna con la ciudad. Providencia, tras las primeras intervenciones relacionadas con el Plan de Pasajes Peatonales, reforzó su carácter comercial y ofreció a sus visitantes un recorrido que potenció la dinámica de caminar y "vitrinear". En el mismo espacio se conglomeraban cafés, bares, galerías de arte, tiendas de decoración, el Drugstore, los caracoles comerciales y, por último, boutiques y tiendas de vestuario que ofrecían ropa de una variada gama de precios y calidad. 


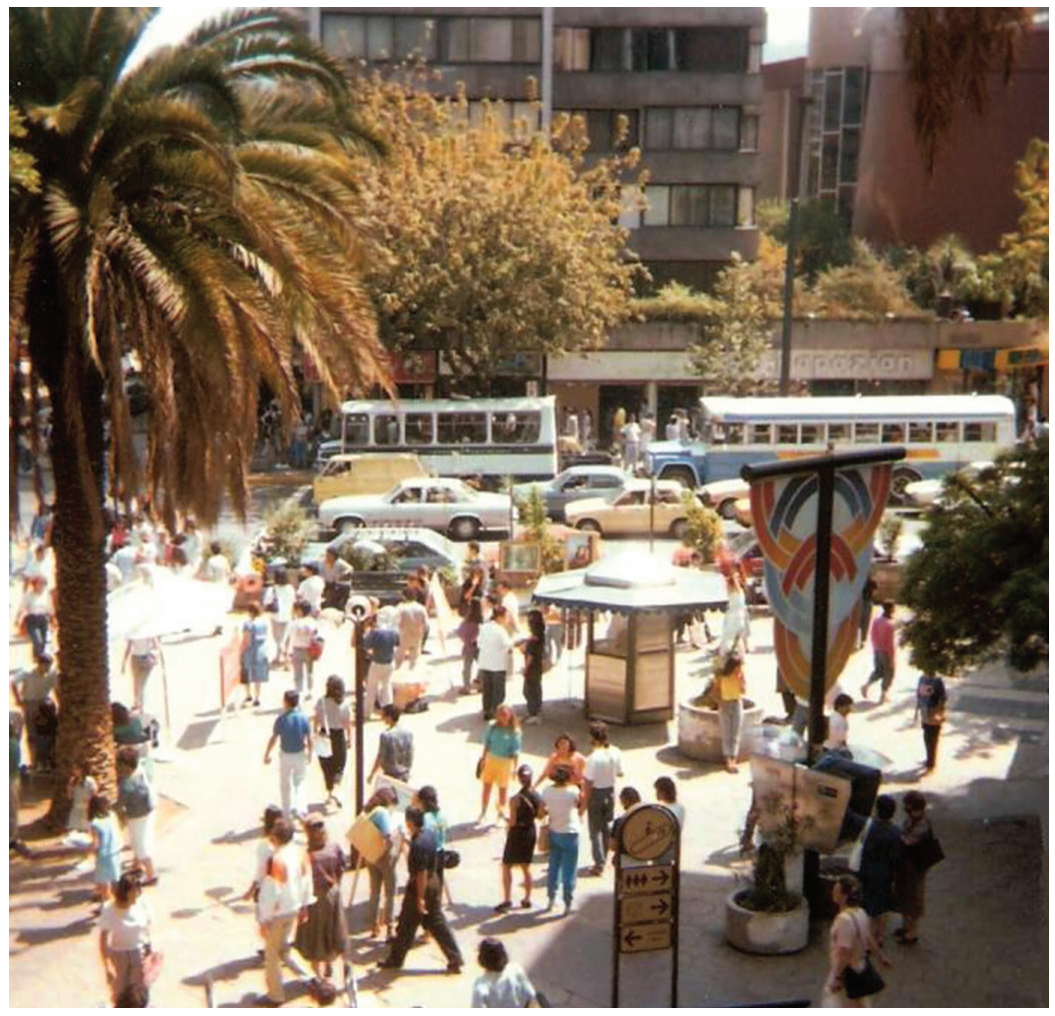

Figura 8. Ricardo Lyon con avenida Providencia en 1980, el mismo año que se inauguró el “Cosmocentro Apumanque" en la comuna de Las Condes. Fuente: enterreno.com.

5. El segundo mall de la ciudad de Santiago fue construido en 1990 en la comuna de La Florida y se llamó: Mall Plaza Vespucio. En 1993 se inaugura el Shopping de La Dehesa y el Mall Alto Las Condes.
Sin embargo, a mediados de los años ochenta, el panorama comenzó a cambiar en Providencia. Muchas de las grandes casas residenciales del sector comercial de la comuna fueron abandonadas para ser demolidas y transformadas en edificios. Esto provocó que, a finales de la década, una gran población flotante llegara a la comuna y transformara las antiguas dinámicas del lugar. Los locales comerciales se diversificaron para responder a las exigencias de quienes ahí trabajaban, y así proliferaron negocios de primera necesidad que despojaron de exclusividad al centro comercial.

De esta manera, emergieron nuevos centros de comercio en los sectores $\mathrm{El}$ Golf, Alonso de Córdova, Presidente Riesco y El Bosque, los cuales se transformaron en espacios de consumo que acogieron a una nueva generación de boutiques. Este fenómeno fue acompañado por el surgimiento de nuevas tiendas y la llegada de exclusivas marcas internacionales. Los enclaves comerciales de la capital se dispersaron y trasladaron a las periferias de la ciudad, lo que provocó una segmentación de los consumidores 5 . Los nuevos lugares de compra dedicados a un público privilegiado no lograron conformar una red de boutiques y tiendas de vestuario que estuviese complementada con otros servicios que favorecieran la estadía de sus consumidores. De esta manera, la sociabilización quedó contenida exclusivamente en el espacio privado y cerrado de las tiendas. Así, ante la crisis y la escasez de los espacios públicos en el Santiago de los ochenta, los herméticos centros comerciales -el mall y las aisladas tiendas en sectores exclusivos de la capital-, aparecen como lugares amables y seguros, condiciones ideales para el ocio y las nuevas dinámicas de consumo. 


\section{Conclusiones}

El desarrollo de la moda moderna en la ciudad de Santiago coincide con una etapa de grandes transformaciones socioculturales vinculadas a las mujeres y a los jóvenes. Ambos encontraron mayores instancias de participación en la vida pública, tanto a nivel país como internacionalmente. Uno de los espacios urbanos que se constituyó como tal fue el nuevo centro comercial de la comuna de Providencia, donde se vivenció la llegada del modelo internacional de producción conocido como prêt-à-portery el emplazamiento de un conjunto de inéditos locales comerciales, las boutiques.

La migración del prêt-à-porter desde Europa a nuestro país estuvo caracterizada por un proceso de adecuación de esta tendencia a la realidad local. Esta transformación se articuló en la premisa del "hacer con menos", adaptando a la escala chilena un fenómeno internacional. Si bien al revisar las revistas femeninas de la época se puede reconocer una moda que se ajusta a las tendencias extranjeras, el proceso detrás de las prendas de vestuario da cuenta de la distancia entre el original y la copia. El prêt-à-porterchileno no fue un fenómeno de masas producido en serie, sino que se significó por la confección a baja escala, a veces artesanal, que aún privilegiaba la exclusividad ante la accesibilidad. En la misma línea se desarrolló su comercialización, liderada por los dueños de las boutiques (en su mayoría mujeres), quienes personalmente activaron la socialización entre su privilegiada clientela.

El centro comercial de Providencia no solo estuvo caracterizado por la compra y venta, sino también por modernas dinámicas de interacción social. Las características de este paisaje comercial favorecieron el recorrido a pie por las calles del sector, por lo que el fenómeno de la moda no solo fue experimentado por los compradores que adquirieron prendas de vestuario en las boutiques, sino que también existió un colectivo (Vaccari, 2008); una puesta en común mantenida por todos los otros actantes presentes, que percibieron la moderna oferta del prêt-à-portersignificado en este inédito conjunto de boutiques y pequeñas tiendas de vestuario. De acuerdo a lo anterior, podemos concluir que en el centro comercial de Providencia se configuró una geografía de la moda. David Gilbert define este concepto como algo más que una serie de calles y comercio dedicado a la venta de prendas y objetos de lujo; son lugares donde la moda es exhibida, observada, imitada y transformada por quienes viven y siguen su vorágine (Gilbert, 2000; Cruz, 2018).

En consecuencia, el declive del centro comercial de Providencia, en cuanto al consumo de moda, está íntimamente relacionado con el surgimiento de nuevos subcentros en el sector Oriente donde los hábitos de compra se diseñaron de manera radicalmente distinta. El sector de Providencia, entre Pedro de Valdivia y Los Leones aproximadamente, conformaba un recorrido en el cual el peatón podía visitar distintos lugares obviando el uso del automóvil. La comercialización de prêt-à-porter en este espacio estuvo íntimamente relacionada con la interacción social y la escenificación, observación y comparación de sus visitantes, dinámicas que fueron facilitadas por el caminar. El concepto del consumidor paseante o flâneur, que estuvo presente en las galerías comerciales del centro de Santiago y después se trasladó al sector comercial de Providencia, quedó obsoleto frente a las nuevas dinámicas de compra que ofreció la década de los noventa, por un lado, con los cerrados malls con marcas de retail y por otro, con exclusivas y alejadas tiendas en "barrios que no se caminan" (L. Fernández, comunicación personal, 23 de mayo de 2017). 


\section{Agradecimientos}

A Macarena García y Pedro Álvarez por su colaboración en el proyecto.

\section{Referencias}

Bannen, G. (1993). “El Comercio en Providencia”, en Revista CA: revista oficial del colegio de arquitectos de Chile, $\mathrm{N}^{\circ} 72$, Santiago.

Barros Cajdler, D. (2013). Estatización de la industria textil durante el Gobierno de la Unidad Popular (1970-1973), Memoria para optar al grado de Licenciado en Ciencias Jurídicas. Facultad de Derecho. Universidad de Chile.

Cerrillo, L. (2010). La moda moderna: Génesis de un arte nuevo. Madrid: Siruela.

CORFO. División de Industrias Textiles y de Vestuario. (1974). Pre-diagnóstico de la industria textil chilena, Santiago. Recuperado de: http://www.memoriachilena. cl/602/w3-article-62484.html

Correa S.; Figueroa C.; Jocelyn-Holt, A., Rolle C. y Vicuña. (2001). Historia del siglo XX chileno. Santiago: Sudamericana.

Crewe, L. (2017). The Geographies of Fashion: Consumption, Space, and Value. Londres: Bloomsbury.

Cruz, W. 2018. La geografía de la moda, Medellín 1900- 1950. En M. Blanco (Presidencia). $4^{\circ}$ Congreso Internacional de Moda y Diseño, Universidad Politécnica de Madrid, Madrid, España.

De Simone, L. (2015). Metamall: Espacio urbano y consumo en la ciudad neoliberal chilena. Santiago: RIL Editores.

De Simone, L. (2015). "Instalando la ciudad del consumo: El palimpsesto urbano del primer shopping mall chileno en el fundo San Luis, Santiago", en Revista Eure, $\mathrm{N}^{\circ} 133$, Santiago.

Dussaillant, J., (2011). La gran tienda: La mujery la ropa hecha en la modernización del comercio minorista de Santiago 1880-1930. Santiago: Ediciones UC.

Errázuriz, L. y Leiva, G. (2012). El Golpe estético: Dictadura militar en Chile 1973-1989. Santiago: Ocho libros.

Frías, P. (Ed). (1987). Industria textil y del vestuario en Chile: Evolución económica y situación de los trabajadores. Santiago: Colección Estudios Sectoriales.

Gilbert, D. (2000). “Urban Outfitting: The City and the Spaces of Fashion Culture", en Fashion Cultures: Theories, Explorations, and Analysis. Londres: Routledge.
Latour, B. (1998). "La tecnología es la sociedad hecha para que dure". En M. Domènech y F. J. Tirado (Comps.), Sociología simétrica. Ensayos sobre ciencia, tecnología y sociedad (pp. 109-141). Barcelona: Gedisa. Lipovetsky, G. (2009). El imperio de los efímero: La moda y su destino en las sociedades modernas. Barcelona: Anagrama.

Miller, D. (Ed.). (1998). Shopping, Place and Identity. Londres: Routledge.

Montalva, P. (2015). Morir un poco: Moda y sociedad en Chile 1960-1976. Santiago: Catalonia.

Mora, R., Zapata, I. (2004). “Comercio, espacio público y gestión local: El caso de Providencia en Santiago de Chile", en Revista de Urbanismo, № 9 , Universidad de Chile, Santiago.

Potvin, J. (2009). The Places and Spaces of Fashion, 18002007. Nueva York: Routledge.

Salinas, J. (2014). Linda, regia, estupenda: Historia de la moda y la mujer en Chile. Santiago: El Mercurio-Aguilar.

Schlack, E. (2001). “Producción privada de espacio público: Espacios Privados de uso público y la planificación por incentivos", en Revista de Arquitectura de la Universidad de Chile, $\mathrm{N}^{\circ} 24$, Santiago.

Squicciarino, N. (1998). El vestido habla. Madrid: Cátedra.

Vaccari, A. (2008). "Reensamblar lo social: Una introducción a la teoría del actor-red”, en Revista CTS, núm. 11, Buenos Aires.

Vidal, J. (2017). Moda al paso: Historia de las boutiques y pequeñas tiendas de vestuario de la zona Oriente de Santiago (1967-1887), Tesis para optar al Título Profesional de Diseñadora, Pontificia Universidad Católica de Chile, Santiago.

Vidal, J., \& Álvarez, P. (mayo de 2018), “El fenómeno del prêt-à-portery las boutiques en Santiago de Chile". En M. Blanco (Presidencia). $4^{\circ}$ Congreso Internacional de Moda y Diseño, Universidad Politécnica de Madrid, Madrid.

Vitale, P. y Vitale A. (comp.). (2000). Los cuerpos dóciles. Buenos Aires: La Marca. 